\title{
Design and Implementation of Automatic Control System for Rice Seed Tape Winding Units
}

\author{
Hongguang Cui, Wentao Ren", Benhua Zhang, Yi Yang, Lili Dai, and Quanli Xiang \\ College of Engineering, Shenyang Agricultural University, \\ Shenyang, 110866, P.R. China \\ cuihongguang7763@163.com, renwentao1958@yahoo.cn, \\ benhuazhang@163.com, tianya@163.com, dadailili@163.com, \\ xiangquanli1020@163.com
}

\begin{abstract}
In order to adapt to the requirements of the development for precision agriculture technology, making the rice seed tape planting technology has been widely used. The paper-making improved the design on the rice seed tape twisting machine especially the seed tape winding units. It proposed seed tape winding automatic control system based on STC90C514AD to realize the rotating speed adjustment of the seed tape disk, used an angular displacement sensor real-time detecting on the disk diameters during the winding process. The system can make the rotating speed reduce when the diameters increase. The experimental results show that the machine performs well. Coefficient of variation of the seed rope linear velocity was $4.64 \%$, the tension uniformed during the seed tape winding process. The working efficiency of the machine is $360 \mathrm{~m} / \mathrm{h}$. It shows that the machine and the control system meet the overall design requirements well.
\end{abstract}

Keywords: The rice seed tape, breaking force of seed tape, winding machine, sensor detection.

\section{Introduction}

The rice direct sowing technology with seed tape based on non-woven (abbreviation is the rice direct sowing technology with seed tape), was a new technology of rice direct sowing proposed in recent years. The technology works into two steps. Firstly, it drops the rice and other materials in PLA non-woven, makes up the seed tape disk in factories. Secondly, it lay the rice seed tape in the ditches according to the requirements of agriculture by using the direct sowing machine which work ditch, lay the seed tape, over the earth and repression in one time. This technology could realize the working of the rice seed tape twisting unrestrict of the soil and other conditions in space, unconstrain of the farming season in time. It has the effect of cost-saving and efficiency-increasing, that can realize simplification and lightness on the works in the fields [1], [2].

* Corresponding author

D. Li, Y. Liu, and Y. Chen (Eds.): CCTA 2010, Part IV, IFIP AICT 347, pp. 428-436, 2011.

(C) IFIP International Federation for Information Processing 2011 
Wentao Ren and his group studied this technology since 2005, had achieved preliminary results. The machine that the group manufactured could make up the seed tape disk [3]. It has the advantages of simple structure, low cost and easy operation. But the seed tape breaks easily and the hill distances accuracy is lower by using this machine during the producing because of the constant rotating speed of the seed tape disk, while the diameters of the disk increasing continuously and the tensions on the seed tape are bigger and bigger. Jun Zhou and his group studied a machine for producing precision seed tape with paper for rice direct sowing based on PLC controller and five motor-driven [4], [5]. The hill distances, the numbers of seeds per hill and the tensile strength of the seed tape improved. However, the machine has disadvantages of high cost and single material twisting. Because of the motor and the shaft for winding moved up and down with the under beam, there is a large inertia force, so it causes uniformity of the twisting on the winding disk.

According to the problems existed, this paper has improved the design on the rice seed tape twisting machine, particularly the winding units of it. It designs a two-way spiral camshaft driven by the seed tape disk shaft, to realize seed tape uniform reciprocating winding. It proposes the automatic control system based on STC90C514AD to realize the rotor speed adjustment of the seed tape winding disk, apply an an gular displacement sensor real-time detecting the disk diameters during the winding process.

\section{Materials and Methods}

It selects spun-bonded non-woven fabric made of polylactide (PLA) as the tape material with width $20 \mathrm{~mm}$ and density $40 \mathrm{~g} / \mathrm{m}^{2}$, which produced in the Jiangyin gaoxin chemical fiber CO., LTD. In Shenyang area, the parameters of the rice seed tape direct sowing technology are: the rice variety is Shendao 7 , amount is about $0.03 \mathrm{~g}$ per hill, seeds distance is $30 \mathrm{~mm}$, the fertilizer selected is granular DAP, amount is about $0.04 \mathrm{~g}$ per hill, the distance between the fertilizer and the seed is $15 \mathrm{~mm}$. The theoretical linear velocity of the rice seed tape is $0.1 \mathrm{~m} / \mathrm{s}$, the twisting numbers of the seed tape is 67 per meter. In order to get convenient storage and realization of direct sowing mechanize operated in field, the disk of the twisting should be standard (inner diameter is $100 \mathrm{~mm}$ and outer diameter is $310 \mathrm{~mm}$ ), seed tape uniform cycloidal winding in disk.

\subsection{Working Principle of the Rice Seed Tape Twisting Machine}

The working principle of the rice seed tape twisting machine is shown in Figure 1. The hardware of the machine consists of non-woven feeding mechanism, binder brushing mechanism, seed sowing mechanism, fertilizer sowing mechanism, materials locating mechanism, twisting mechanism, drying mechanism, seed tape disk winding mechanism, seed tape fracture testing mechanism and so on. The software of the machine consists of MCU control system, computer monitoring and recording system and so on. 
Non-woven has been made in disk shape (1) by its manufacturer, pulled by the winding mechanism and passed through non-woven rollers (2), so that the non-woven is flat to avoid distort or wrap. During the non-woven went on continuously, it passes binder brushing mechanism $(3,4,5)$ covered, the binder machine is driven by binder motor (5), the binder is made by modified maize starch [4]. The non-woven with binder over it enters into the $\mathrm{V}$-supporting board (25) and becomes the V-cross section, which can carry seeds and fertilizers that ensure they will not fall off from the non-woven.

Rice seeds and fertilizers dropped in the holes of the synchronous belt (24) by seeding from the horizontal plate seed metering device (7) driven by motor (6) and cell wheel feed for fertilizer (10) driven by motor (11), through the seed transmitting tube (21) and the fertilizer transmitting tube (22) respectively. After the synchronous belt has been carried on, they have the same linear velocity with non-woven, at that time the materials dropped on the non-woven passed by through the hole of the Vsupporting board (25).

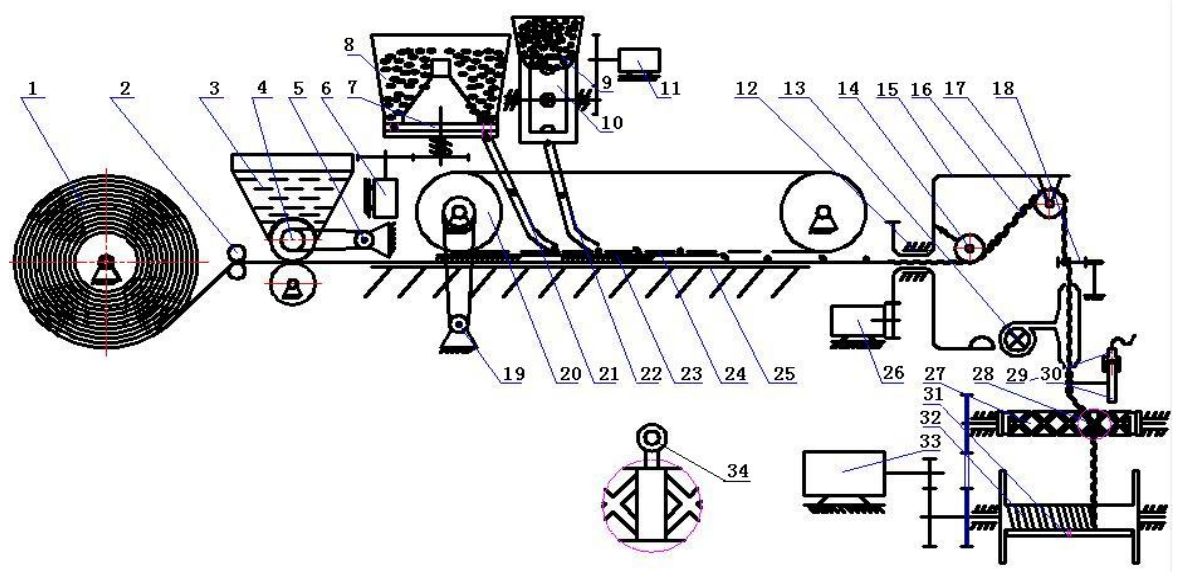

1 non-woven disk ; 2 non-woven rollers ; 3 binder ; 4 binder brushing rollers ; 5 motor for binder ; 6 motor for seed metering device ; 7 horizontal plate seed metering device ; 8 rice seeds box ; 9 fertilizers box ; 10 cell wheel feed for fertilizer ; 11 motor for fertilizer ; 12 bearing of U-rotating frame ; 13 hair dryer ; 14 seed supporting roller ; 15 U-rotating frame ; 16 rice seed tape ; 17 seed tape steering roller ; 18 steering cylinder ; 19 motor for secondary seeding ; 20 secondary seeding driving pulley ; 21 seed transmitting tube ; 22 fertilizer transmitting tube ; 23 secondary seed supporting board ; 24 secondary seeding synchronous belt with holes ; $25 \mathrm{~V}$-supporting board ; 26 stepper for U-rotating frame ; 27 two-way spiral camshaft ; 28 seed tape guiding block ; 29 photoelectric sensor ; 30 seed tape break detection device ; 31 diameters of seed tape disk detection device ; 32 seed tape disk ; 33 step motor for the seed tape disk ; 34 seed tape guiding ring

Fig. 1. Working principle of the rice seed tape twisting machine 
The parts of the non-woven which carried seeds and fertilizers come into being the seed tape had twisting numbers by the action of the U-rotating systems $(12,14,15$, 17) driven by motor (26) and the steering cylinder (18). The hair dryer (13) dries the binder in the tape. And then the seed tape passes though the guiding ring (34), in the action of the two-way spiral camshaft (27) driven by the seed tape disk shaft. Eventually the seed tape winding uniform on the disk with the space required. And then the seed tape winding on the disk (32) has driven by step motor (33) which in the automatic control system that shown in Figure 2 and Figure 3.

\subsection{Analysis of the Parameters of the Automatic Control System}

As it is shown in Figure 1, during the seed tape winding, the diameters of the seed tape disk increasing when the turn numbers of the seed tape increasing. In order to obtain a stable linear velocity of the seed tape, the automatic control system has been designed, which consists of seed tape disk diameters detection device (31) and STC90C514AD. It changes the rotating speed of step motor (33), realizes the rotating speed of the disk reducing when the diameters increase. It avoids the seed tape broken because of the seed tape linear velocity constant, and assures the tensions uniform in the process of the seed tape rolling.

The working principle of the automatic control system for linear velocity of the seed tape is shown in Figure 2. It consists of U-rotating twisting mechanism, seed tape winding guiding mechanism, seed tape breaking detection mechanism, automatic control system for the seed tape disk winding and so on. The automatic control system for the seed tape disk winding consists of seed tape disk radius detecting roller, seed tape disk radius detecting pendulum, angular displacement sensor, MCU, step motor driver and the seed tape disk.

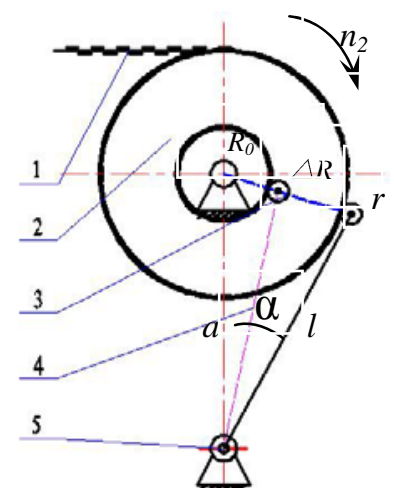

1 seed tape ; 2 seed tape disk ; 3 seed tape disk radius detection roller ; 4 seed tape disk radius detecting pendulum ; 5 angular displacement sensor

Fig. 2. The principle of the automatic control system for linear velocity of the seed tape 
During the process of the seed tape winding, the calculation model of the seed tape linear velocity on the disk is,

$$
v=\left(R_{0}+\Delta R\right) \cdot \frac{2 \pi \cdot n_{2}}{60}
$$

In the formula (1), $v$-the linear velocity of the seed tape, $\mathrm{m} / \mathrm{s} . R_{0^{0}}$-initial radius of the seed tape disk, m. $\triangle R$-increment for the radius of the seed tape disk, m. $n_{2}$-rotating speed of the seed tape disk, $\mathrm{r} / \mathrm{min}$.

It can be known from the formula (1) that, only when the rotating speed $\left(n_{2}\right)$ of the seed tape disk meets the formula $n_{2}=R_{0} \cdot n_{0} /\left(R_{0}+\Delta R\right)$, the linear velocity of the seed tape is stable and the tension of the seed tape is uniform $\left(n_{0}\right.$-initial rotating speed of the seed tape disk, r/min). Therefore, the key of the design is accuracy and timely detection of the disk radius and turning them into electrical signals to transmit to the control system.

By applying DS36-V/A angular displacement sensor to detect the seed tape radius $(R+\triangle R)$, using the system calibration, the regression equation of the seed tape disk radius is,

$$
V=0.057 \alpha-0.035
$$

In the formula (2), $V$-output voltage of the angular displacement sensor, $\mathrm{V} . \alpha$ angular of the radius detecting pendulum, ${ }^{\circ}$.

Analysis of variance results shows that the equation regression coefficient $R>0.95$. It shows that the detection system has good linearity and high precision.

The relationship between the angular of the radius detecting pendulum and the radius of disk is,

$$
\cos \alpha=\frac{a^{2}+l^{2}-\left(R_{0}+\Delta R+r\right)^{2}}{2 a l}
$$

In the formula (3), $a$-centre distance of the angular displacement sensor and the seed tape disk, $\mathrm{m}$. $l$-length of the radius detecting pendulum, $\mathrm{m}$. $r$-radius of the seed tape disk radius detecting roller, $\mathrm{m}$.

It uses 57BYGH306 step motor and DL-023MDC step motor driver to achieve the rotating speed adjustment of the seed tape disk. The rotating speed of the step motor depends on the pulse frequency, step angle depends on the microstep of the step motor driver. When the step motor driver in the input pulse of $200 \mathrm{~Hz}$, it is in the concussion zone, easy to damage the internal components. So apply pulse of $350 \mathrm{~Hz}$ as the low frequency starting point. In order to satisfy the step motor driver input pulse, it set transmission ratio of $4: 1$ (that is $n_{2} / n_{1}=4 / 1$ ) gear-driven to the seed tape disk. It has,

$$
n_{1}=\frac{60 \cdot \theta \cdot f}{360^{\circ}}
$$


In the formula (4), $\theta$-step angle of step motor, ${ }^{\circ} . f$-pulse frequency of step motor driver, HZ. $n_{l}$ - rotating speed of step motor, $\mathrm{r} / \mathrm{min}$.

\subsection{Working Principle of the Automatic Control System for Linear Velocity of the Seed Tape}

Working principle of the automatic control system for linear velocity of the seed tape is shown in Figure 3. It is a control system of the constant value. Initial value of the linear velocity $\left(v_{0}\right)$ is set from the keyboard of the MCU. Through the MCU operating, it drives the step motor work in the rotating speed of $n_{0}$ and produces an initial voltage value $(V)$. Compare $V$ with the $V_{t}$, which is converted from the feedback device of the seed tape radius detection, and get $\triangle V$. After AD conversion, amplification and calculation of the MCU, it outputs frequency $(f)$ to step motor driver to change the rotating speed of the step motor, adjusts the rotating speed of the seed tape disk, outputs actual linear velocity $\left(v_{m}\right)$ of the seed tape. The radius of the seed tape disk $(R)$ increased, when the numbers of the seed tape winding on the disk increasing. The value of $R$ is detected by the feedback device of the seed tape radius detection to output voltage value $\left(V_{t}\right)$ and compared with initial voltage value to complete the process of the automatic control system. The automatic control system communicates with host computer through the RS232 bus to realize data acquisition, storage, and comparison analysis and so on.

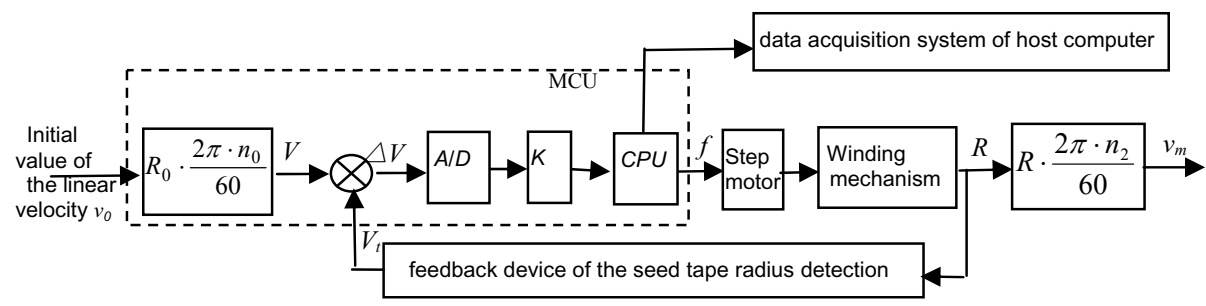

Fig. 3. Working principle of automatic control system for linear velocity of seed

\section{Results and Discussion}

The prototype test of the hardware system has been done to verify the accuracy of the automatic control system. Contrast curves of the theoretical rotating speed and actual rotating speed of the seed tape disk are shown in Figure 4.

It can be seen from Figure 4 that the rotating speed of the seed tape disk present ladder form decreases with the time increasing during the winding process. The reason is that the rotating speed of the seed tape disk decreases with the seed tape disk radius increasing, while the radius of the seed tape disk increases only after the seed tape winding fully one layer on the disk, which changes the equivalent to a step signal. The time of one layer spending is different in a constant linear velocity, so the changes of the rotating speed of the seed tape disk and the time into the relationship of ladders. 


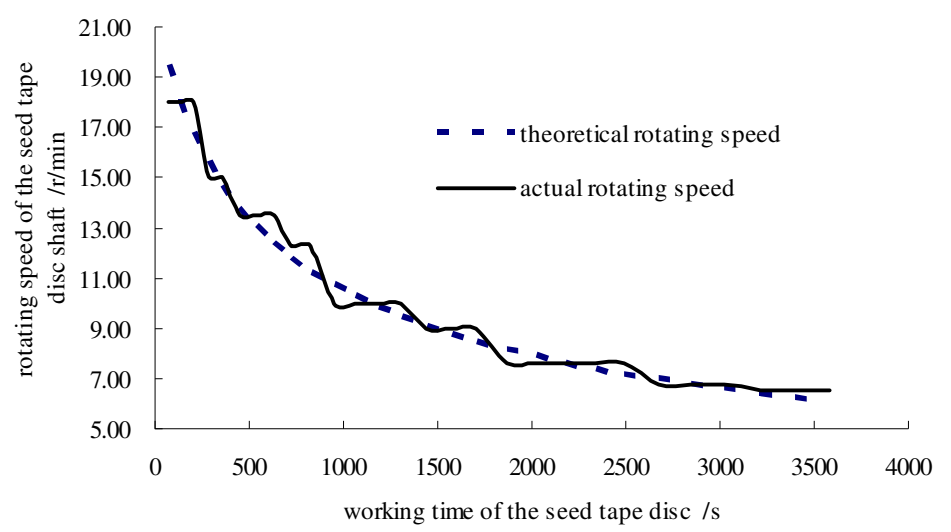

Fig. 4. Contrast curves of theoretical and actual rotating speed of the seed tape disk

Descriptive statistics of the actual seed rope linear velocity has been listed in table 1. After comparative analysis with the theoretical linear velocity $(0.1 \mathrm{~m} / \mathrm{s})$, the changes of the actual linear velocity is little, it meets the requirements of design.

Table 1. Descriptive statistics of the actual seed rope linear velocity

\begin{tabular}{ccccc}
\hline $\begin{array}{c}\text { Linear } \\
\text { velocity mean } \\
/ \mathrm{m}\end{array}$ & $\begin{array}{c}\text { Std. } \\
\text { deviation } \\
/ \mathrm{m}\end{array}$ & Minimum & Maximum & $\begin{array}{c}\text { Coefficient } \\
\text { of variation } \\
1 \%\end{array}$ \\
\hline 0.101 & 0.0047 & 0.092 & 0.109 & 4.64 \\
\hline
\end{tabular}

\section{Conclusions}

(1) It has designed rice seed tape twisting machine in this paper. The machine consists of non-woven feeding mechanism, binder brushing mechanism, seed sowing mechanism, fertilizer sowing mechanism, materials locating mechanism, twisting mechanism, drying mechanism, seed tape disk winding mechanism, seed tape fracture testing mechanism and so on. The machine can drop seeds and fertilizers on the spunbonded non-woven fabric made of polylactide (PLA) and made into seed tape disk.

(2) It has designed a two-way spiral camshaft driven by the seed tape disk shaft to realize seed tape reciprocating winding in the disk, when the diameters of the disk bigger, the rotating of the spiral camshaft slower, which ensures the seed tape disk uniformity of winding. That realizes the constant speed of the seed tape.

(3) It has studied an automatic control system for linear velocity of the seed tape in this paper. The system consists of seed tape disk radius detecting roller, seed tape disk radius detecting pendulum, angular displacement sensor, MCU, step motor driver and the seed tape disk. It overcomes the shortcomings of the first-generation prototype such as the seed tape breaking easily because the diameters of the disk increase continuously and the tensions on the seed tape are bigger and bigger. The experimental results show that the machine performs well. The actual rotating speed slowed in 
ladder figure when the diameters of the disk increased. Analysis by the SPSS, the linear velocity mean was $0.101 \mathrm{~m} / \mathrm{s}$, the Std. deviation was 0.0047 , the coefficient of variation of the actual seed rope linear velocity was $4.64 \%$. It has no fracture during the process of twisting working, it meets the design requirements. The system also has advantages of low cost and suitable for promotion and application.

\section{References}

1. Lv, X.R., Ren, W.T.: Analysis and Research for the Technology of Rice Direct Sowing with Seed Rope. Journal of Agricultural Mechanization Research 1, 212-215 (2008) (in Chinese)

2. Ren, W.T., Li, X.S., Cui, H.G.: Effect of the Technigue of Rice Direct Sowing with Seeds Twisted in Paper Rope on Rice Yield Character. Journal of Shenyang Agricultural University 36(3), 265-270 (2005) (in Chinese)

3. Ren, W.T., Li, X.S., Zhang, Y.S.: Development and Experiment on a Rice Seed Rope Twisting Machine. Journal of Agricultural Mechanization Research 6, 169-172 (2005) (in Chinese)

4. Zhou, J., Ji, C.Y.: Development of Machine for Producing Precision Seeding Rope with Paper For Rice Direct Sowing. Transaction of the CSAE 25(7), 79-83 (2009) (in Chinese)

5. Zhou, J., Ji, C.Y.: Machine for Producing Rice Seed Rope and Field Experiment. Journal of China Agricultural University 14(2), 98-102 (2009) (in Chinese)

6. Zhang, T., Ren, W.T., Ma, Y.: Parameter Analysis on Twisting Rope Machine of Rice Seed Rope Twisting Machine. Journal of Agricultural Mechanization Research 12, 78-79 (2006) (in Chinese)

7. Ren, W.T., Lv, X.R., Zhang, B.H.: Dynamic Response of Taped Type Rice Direct Seeding Machine for Field Surface Roughness. Transactions of the Chinese Society for Agricultural Machinery 40(8), 58-61 (2009) (in Chinese)

8. Ren, W.T., Dong, B., Cui, H.G.: Experiment on the Motion Characteristics of Rice Seeds after Collision with Different Slopes. Transactions of the CSAE 25(7), 103-107 (2009) (in Chinese)

9. Ren, W.T., Yang, Y., Zhang, B.H.: Design and Implementation of Automatic Control System for Sectional Type Subsurface Drip Irrigation in Greenhouse. Transactions of the CSAE 25(8), 59-63 (2009)

10. Ren, W.T., Dai, L.L., Cui, H.G.: Effect of Modified Maize Starch Binder on the Quality of Seed Tape Twisting. Transactions of the CSAE 26(5), 164-169 (2010) (in Chinese)

11. Luo, X.W., Liu, T., Jiang, E.C.: Design and Experiment of Hill Sowing Wheel of Precision Rice Direct-seeder. Transactions of the CSAE 23(3), 108-112 (2007) (in Chinese)

12. He, R.Y., Luo, H.Y., Li, Y.T.: Comparison and Analysis of Different Rice Planting Methods in China. Transactions of the CSAE 24(1), 167-171 (2008) (in Chinese)

13. Guo, Y.X., Liu, W.N., Zhao, Q.X.: Study on Microprocess Control System Based on Precise Sowing. Journal of Agricultural Mechanization Research 9, 81-83 (2008) (in Chinese)

14. Qiao, X.J., Shen, Z.R., Chen, Q.Y.: Design and Realization of General Computer Monitoring and Controlling System for Environment of Agricultural Facilities. Transactions of the CSAE 16(3), 77-80 (2000) (in Chinese)

15. Wu, Q.H., Xu, B.Q.: Analysis of Synchronized Control System for Multi-Motor. Autocontrol O.I.Automation 22(1), 20-24 (2003) 
16. Kevin, P.: Synchronized Motion Control withthe Virtual Shaft Control Algorithm and Acceleration Feedback. In: Proceedings of the American Control Conference, American, pp. 2102-2106 (1999)

17. Anderson, R.G., Meyer, A.J., Valenzhuela, M.A.: Web Machine Coordinated Motion Control Viaelectronic line-Shafting. IEEE.Trans. Ind. Application 37(1), 247-254 (2001)

18. Xu, H.B., Du, X.B.: Control System Design for Stepper Motors. Journal of Chongqing Institute of Technology (Natural Science) 22(5), 32-34 (2008)

19. Zhong, Y.S., Yang, J.Q., Deng, J.L.: Multivariable Fuzzy Control of Temperature and Humidity in a Greenhouse. Transaction of the Chinese Society for Agricultural Machinery 32(3), 75-78 (2001) (in Chinese)

20. Gong, C.K., Chen, C.Y., Mao, H.P.: Multivariable Fuzzy Control and Simulation of a Greenhouse Environment. Transaction of the Chinese Society for Agricultural Machinery 31(6), 52-54 (2000) (in Chinese)

21. Ding, W.M., Wang, X.C., Li, Y.N.: Review on Environmental Control and Simulation Models for Greenhouse. Transaction of the Chinese Society for Agricultural Machinery 40(5), 162-168 (2009) (in Chinese)

22. Yu, Y.C., Hu, J.D., Mao, P.J.: Fuzzy Control for Environment Parameters in Greenhouse. Transaction of the Chinese Society of Agricultural Engineering 18(2), 72-75 (2002) (in Chinese) 